LIAMES 15(2): 355-374 - Campinas, Jul./Dez. - 2015

\author{
Lorena Cayré Baito \\ (Consejo Nacional de Investigaciones \\ Científicas y Tecnológicas, \\ CONICET/Universidad \\ Nacional del Nordeste, UNNE, Argentina)
}

\title{
Una primera aproximación a la variación vocálica inter-dialectal en wichí*
}

\begin{abstract}
Scholars agree that Wichí language (Mataco-mataguayan family) has a five vowel system /a/, /e/, /i/, /o/ and /u/. However one of the most salient differences among Wichí dialects is the following vowel variation: $h a^{\prime} l \boldsymbol{o}$ vs. $h a^{\prime} l \boldsymbol{a}$ 'tree'; we'nu vs. we'no 'to give -something to someone-'; hep vs. hup 'house'; ti'tcu vs. te'tfe 'egg'; pejak-hi vs. pejek-hi 'mirror, photo'; tip vs. tup 'nest'. In the literature this variation has been partially analyzed by Terraza (2003) and Messineo \& Braunstein (1990). The aim of this paper is to study this vowel variation between two Wichí dialects, one of them spoken in Ingeniero Juárez (Formosa, Argentina) and the other one, in Tartagal (Salta, Argentina). We consider a diachronic explanation for this phenomenon; our hypothesis is that current vowel variation is the result of a chain shift that combines both raising and fronting of vowels (back vowels move to the front).
\end{abstract}

KEYWORDS: Wichi; Wichi dialects; Vowel variation; Vowel chain shift.

RESUMO: Os estudiosos concordam que a língua wichí (família mataco-mataguaya) tem um sistema de cinco vogais /a/,/e/,/i/,/o/e /u/. Contudo, uma das principais diferenças entre os dialetos do wichí é a seguinte variação vocálica: $h a^{\prime} l \boldsymbol{l}$ vs. $h a^{\prime} l \boldsymbol{a}$ 'árbol'; we'nu vs. we'no 'dar -alguma coisa a alguém-'; hep vs. hup 'casa'; ti'tcu te'tse 'huevo'; pejak-hi vs pejek-hi 'espelho, foto'; $i p$ vs. Iup 'nido'. Na literatura, essa variação tem sido parcialmente estudada por Terraza (2003) e Messineo e Braunstein (1990). O objetivo deste artigo é analisar a variação vocálica entre dois dialetos do wichí, um falado em Ingeniero Juárez (Formosa, Argentina) e o outro, em Tartagal (Salta, Argentina). Consideramos uma explicação diacrônica para este fenômeno; nossa hipótese é a de que a variação da vogal atual resulta de uma mudança em cadeia que combina alçamento e movimento das vogais posteriores para vogais anteriores.

PALAVRAS CHAVES: Wichí; Dialetos do wichí;Variação vocálica; Mudança vocálica em cadeia.

* Deseamos agradecer al profesor Dr. Marco Antônio Oliveira por sus valiosas observaciones y comentarios al respecto de este trabajo y a Sergio Merino, Luisa Tolapa y Jonás Barbieri por estar siempre dispuestos a enseñarnos su lengua. 


\section{Introducción}

La lengua wichí es hablada en la región conocida como el Gran Chaco, una llanura extensa de aproximadamente $600.000 \mathrm{~km}^{2}$ que abarca los países de Argentina, Bolivia y Paraguay (Braunstein y Miller 1999) ${ }^{1}$ y en la que viven alrededor de 40 pueblos originarios que hablan no menos de 29 lenguas con diferentes niveles de vitalidad (Censabella 2009a: 145). Tradicionalmente esta región se divide en tres regiones: Chaco Boreal, Chaco Central y Chaco Austral. El Chaco Boreal se extiende desde el río Pilcomayo hasta la planicie del Mato Grosso; el Chaco Central se delimita entre los ríos Pilcomayo y Bermejo y el Chaco Austral, se extiende desde el río Bermejo hasta inmediaciones del río Salado. Las comunidades indígenas wichís habitan la zona del Chaco central (véase figura 1) que comprendería el norte de la República Argentina (en las provincias de Chaco, Formosa y Salta) y una pequeña porción limítrofe de la República de Bolivia (Censabella 2009a: 145).

El wichí se caracteriza por presentar una diversidad dialectal muy rica y compleja que supera la tradicional división de esta lengua en tres dialectos: noctén, vejoz y guisnay. Esta división fue inicialmente propuesta por Najlis $(1968)^{2}$ y luego retomada por Tovar (1984). Tovar (1984) usa la denominación noctén para referirse a los hablantes de las ciudades bolivianas de Yacuiba, Villa Montes y Crevaux; vejoz para los hablantes que residen en las zonas de Tartagal y el Río Bermejo y guisnay para denominar a los que habitan la ribera derecha del río Pilcomayo, actual frontera argentino-boliviana.

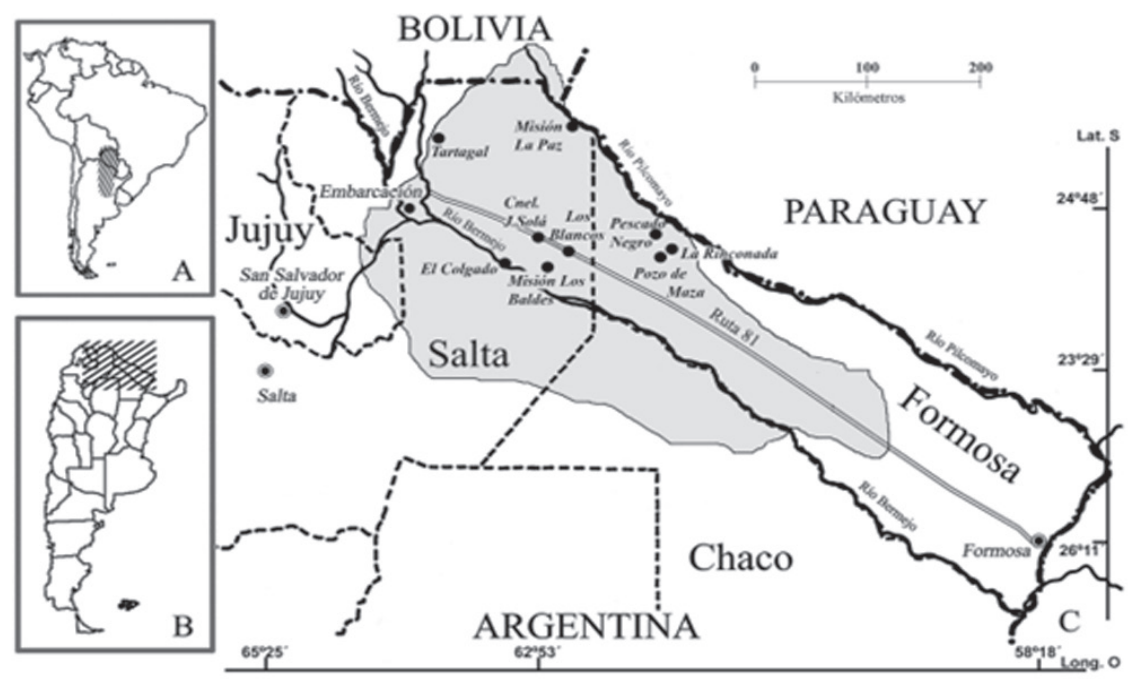

Figura 1. Localización aproximada de los wichís en la región del Gran Chaco. Tomado de Suárez y Arenas (2012).

${ }^{1}$ De acuerdo con Braunstein y Miller (1999: 1-2) la región de el Gran Chaco limita con la precordillera de los Andes, al sur con la cuenca del río Salado, al este con los ríos Paraguay y Paraná y al norte con la meseta del Mato Grosso.

${ }^{2}$ Najlis (1968) propone de manera provisoria, una primera división dialectal de los wichís en cinco parcialidades a partir de un estudio fonológico que ella misma realizó: vejoces (que ocupan el límite oeste), noctenes (que ocupan el norte y extremo oeste), montaraces (que ocupan la zona entre los ríos Bermejo y Pilcomayo), guisnais (que ocupan el norte, parte central y este) y matacos (que ocupan el sur). 
Autores como Gerzenstein (1992) y Messineo y Braunstein (1990) demostraron en sus estudios que los dialectos de la lengua presentan más divisiones. Gerzenstein (1992) identifica una sub-división dialectal en el enclave wichí de las Lomitas (Formosa), que sigue el curso Noroeste-Sudeste (NO-SE) de los ríos Bermejo y Pilcomayo: los phomxleley (los arribeños), para los que viven en el noroeste y los chomxleley (los abajeños) para referirse a los que habitan en el sudeste. Por su parte, Messineo y Braunstein (1990) sostienen que las variaciones lingüísticas son mucho más numerosas. Así, Braunstein (2009) distingue un total de 22 tribus wichí (véase figura 2).

Messineo y Braunstein (1990) sostienen que el carácter divergente de las modalidades lingüísticas puede explicarse por el hecho de que los wichís se organizaban tradicionalmente en tribus o conjuntos de bandas aliadas que nomadizaban cíclicamente en un territorio. Las tribus eran grupos relativamente aislados unas de otras, aunque la relación entre ellas era frecuentemente hostil; esto implicaba una limitación en los intercambios.

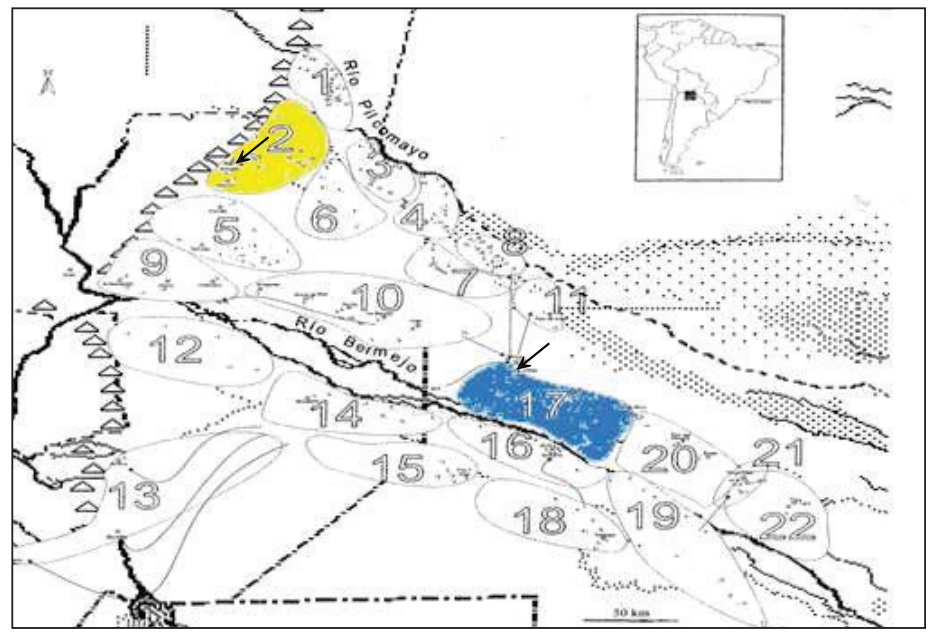

Figura 2. Mapa de las comunidades (tribus) wichí, modificado de Braunstein (2009). Las flechas negras indican la ubicación aproximada de las dos localidades de estudio: Tartagal e Ingeniero Juárez.

\section{Referencias:}

1. Villa monte

2. Mosconi

3. La Paz

4. Santa Teresa

5. Traslado

6. Pozo El Toro1

7. El Chorro

8. Potrillo

9. Embarcación

10. Ruta 81

11. Pozo de Maza

\section{La Unión}

13. Apolinario Saravia

14. Rivadavia

15. Comandancia Frías

16. Sauzalito

17. Juárez (Barrio Viejo)

18. Nueva Pompeya

19. Nueva Población

20. Bazán

21. Las Lomitas (Matadero)

22. Pozo del Tigre 
Actualmente los estudiosos coinciden en considerar al wichí como una gran continuidad dialectal ${ }^{3}$ que abarca una gran extensión, con una franja que comienza desde la mitad del río Bermejo hacia el oeste, con enclaves en ambas orillas, hasta llegar a la provincia de Salta y luego hacia el norte, desde Salta hasta el departamento Tarija en Bolivia (Censabella; Giménez; Gómez 2011). Esta gran diversidad es reconocida por los hablantes mismos quienes manifiestan entenderse unos a otros a pesar de las diferencias. Hasta el momento no existe un trabajo que haya podido sistematizar la compleja diversidad dialectal de esta lengua. ${ }^{4}$

De acuerdo con Censabella et al. (2011) una de las particularidades de la continuidad dialectal es que las variaciones fonéticas, morfo-sintácticas y lexicales son acumulativas. El objetivo de este trabajo es describir y explicar la variación articulatoria entre vocales observada entre el wichí hablado en Tartagal, Salta (figura 2, $\mathrm{N}^{\mathrm{o}}$ 2) y el wichí de Ingeniero Juárez, Formosa (figura 2, $\mathrm{N}^{\mathrm{o}} 17$ ), la cual constituye una de las principales variaciones fonológicas de esta lengua.

La variación vocálica no es exclusiva a estas dos variedades ya que ha sido registrada por los estudiosos entre otras variedades del wichí; por este motivo, la finalidad de este trabajo es brindar una primera explicación diacrónica de este fenómeno de variación vocálica con el propósito de poder seguir indagando en futuros estudios y dar cuenta de su complejidad.

El corpus de datos fue confeccionado en base a tres hablantes. Dos de los hablantes son oriundos del norte de Salta, pero actualmente viven en las comunidades Lapacho 2-3 y Chorote de la ciudad de Tartagal, provincia de Salta. El otro hablante es oriundo de la comunidad Barrio Viejo de la ciudad de Ingeniero Juárez, provincia de Formosa; actualmente vive en Resistencia.

En la sección $\S 2$ describimos la variación vocálica, en $\S 3$ presentamos los antecedentes de análisis en torno a esta temática; en $\S 4$ ofrecemos una breve descripción fonética de las vocales en las variedades estudiadas; en $\$ 5$ brindamos una primera explicación diacrónica sobre este fenómeno de variación vocálica; en $\$ 6$ comparamos nuestro corpus con datos de otras variedades dialectales. Finalmente, en la sección $\S 7$ presentamos las conclusiones.

\footnotetext{
${ }^{3}$ El continnum o continuidad dialectal es un conjunto de dialectos geográficamente contiguos (Censabella et al. 2011).

${ }^{4}$ Los trabajos sobre variación dialectal wichí consisten básicamente en comparaciones entre dos o más variedades del wichí. Entre ellos podemos citar a: 1) Messineo y Braunstein (1990)comparan datos del dialecto bazanero (hablado en la comunidad Juan G. Bazán de Formosa) con el wichí estudiado por Viñas Urquiza (hablado en las comunidades Misión Chaqueña y Misión la Paz de Salta). 2) Gerzeinsten (1992) distingue dos grupos dialectales en el enclave wichí de las Lomitas (Formosa): los phomxleley (los arribeños), para los que viven en el noroeste y los chomxleley (los abajeños) para referirse a los que habitan en el sudeste. Esta diferenciación dialectal fue establecida por la autora al comparar datos fonéticos y fonológicos entre ambos grupos. 3) Fernández Garay y Spinelli (2009) presentan aspectos fonéticos y fonológicos del wichí hablado en Salta (Rivadavia Banda Norte), desde la perspectiva de la sincronía dinámica de la escuela funcionalista francesa, con la finalidad de mostrar la variación existente en el nivel fonético-fonológico. 4) Censabella (2009b) establece y compara, desde la perspectiva funcional y de la sincronía dinámica, el sistema fonológico de un conjunto de variedades, denominadas por la autora variedades orientales del Este y del Oeste. Las variedades de Oeste corresponden a las localidades de El Sauzalito (Chaco) y Teniente Fraga y Misión el Carmen de la provincia de Formosa. Y las variedades del Este, a las localidades Colonia Muñiz y Bazán de Formosa.
} 


\section{Variación articulatoria de vocales}

Entre los estudiosos del wichí hay un acuerdo en que esta lengua tiene un sistema de cinco fonemas vocálicos: dos vocales altas, una posterior $/ \mathrm{u} / \mathrm{y}$ otra anterior $/ \mathrm{i} / ;$ dos vocales medias, una posterior /o/ y otra anterior /e/ y una vocal baja central /a/. Algunos autores que han estudiado el wichí hablado en el norte de Salta (Viñas Urquiza 1974; Fernández Garay y Spinelli 2009) y en Bolivia (Claesson 1994), postulan la existencia de un sexto fonema, la vocal baja posterior /a/.

En nuestro análisis identificamos cinco fonemas vocálicos tanto para Tartagal como para Ingeniero Juárez, no identificamos la vocal baja posterior /a/. El valor distintivo entre estas vocales se puede observar en los siguientes pares mínimos y/o aproximados de la tabla 1 correspondientes a las dos variedades en estudio.

Tabla 1. Ejemplos de pares mínimos y aproximados para demostrar el valor distintivo de las vocales /a/, /e/, /i/, /o/ y /u/ en las dos variedades en estudio.

\begin{tabular}{|c|c|c|}
\hline Oposiciones & Ingeniero Juárez (Formosa) & Tartagal (Salta) \\
\hline /a/ vs. /o/ & ha't'e 'quién' vs. ho'te 'también' & ha'la 'árbol' vs. ho'lo 'arena' \\
\hline /a/ vs. /u/ & łaj 'frutas' vs. łuj 'semillas' & (o)'t'an 'yo grito' vs. t'un 'duro' \\
\hline /e/ vs. /a/ & $\begin{array}{c}\text { u'jel 'yo estoy cansada/o' vs. u'jał } \\
\text { 'yo respiro' }\end{array}$ & ts'ak 'ombligo' vs. tsek 'barrer' \\
\hline /e/ vs. /i/ & łep 'nido' vs. tip 'borde' & tfi'nax 'hierro' vs. t fe'nax 'montaña' \\
\hline /i/ vs. /o/ & x'i'tsax 'malo' vs. x'o'tsax 'víbora' & si'p?a 'policía' vs. so'p?a 'cera' \\
\hline /i/ vs. /u/ & jik 'ir' vs. juk 'afirmar' & łup 'nido' vs. łip ‘borde’ \\
\hline /o/ vs. /i/ & (u)po'ni 'mi garganta' vs. pi'ni 'miel' & jik 'ir' vs. jok 'afirmar' \\
\hline /u/ vs. /a/ & mak 'cosa' vs. muk'w 'polvo' & hun 'lanza' vs. han 'perseguir' \\
\hline$/ \mathrm{u} / \mathrm{vs} . / \mathrm{o} /$ & (i)'mo 'él duerme vs. mukw 'polvo' & hup 'cesped' vs. hokw 'palo santo' \\
\hline
\end{tabular}

Sin embargo, una de las diferencias entre los dos sistemas fonológicos es la variación articulatoria entre vocales, como podemos ver en la tabla 2. En la figura 3 esquematizamos esta variación.

Tabla 2. Variación articulatoria de vocales entre el wichí de Ingeniero Juárez y el wichí de Tartagal.

\begin{tabular}{|c|c|c|c|}
\hline \multicolumn{2}{|c|}{ Ingeniero Juárez (IJ) } & \multicolumn{2}{|c|}{ Tartagal $(\mathrm{T})$} \\
\hline $\begin{array}{l}/ \mathbf{u} / \\
\text { we'nu }\end{array}$ & 'dar' & $\begin{array}{l}/ \mathbf{0} / \\
\text { we'no }\end{array}$ & 'dar' \\
\hline $\begin{array}{l}/ \mathbf{0} / \\
\text { i'tox }\end{array}$ & 'fuego' & $\begin{array}{l}/ \mathbf{a} / \\
\text { i'tax }\end{array}$ & 'fuego' \\
\hline
\end{tabular}




\begin{tabular}{|c|c|c|c|}
\hline $\begin{array}{l}\text { /e/ } \\
\text { pejek-hi }\end{array}$ & 'espejo, foto' & $\begin{array}{l}\text { /a/ } \\
\text { pejak-hi }\end{array}$ & 'espejo, foto' \\
\hline $\begin{array}{l}/ \mathbf{i} / \\
+e^{\prime} t \int e\end{array}$ & 'huevo' & $\begin{array}{l}/ \mathbf{e} / \\
\text { di'tcu }\end{array}$ & 'huevo' \\
\hline $\begin{array}{l}/ \mathbf{i} / \\
\text { tip }\end{array}$ & 'nido' & $\begin{array}{l}/ \mathbf{u} / \\
\text { łup }\end{array}$ & 'nido' \\
\hline $\begin{array}{l}/ \mathrm{e} / \\
{ }^{2} \mathrm{t}^{2} \mathrm{ek}^{\mathrm{w}} \mathrm{e}\end{array}$ & 'buscar' & $\begin{array}{l}/ \mathbf{u} / \\
\mathrm{t}^{2} \mathbf{u} \mathrm{k}^{\mathrm{w}} \mathrm{e}\end{array}$ & 'buscar' \\
\hline
\end{tabular}

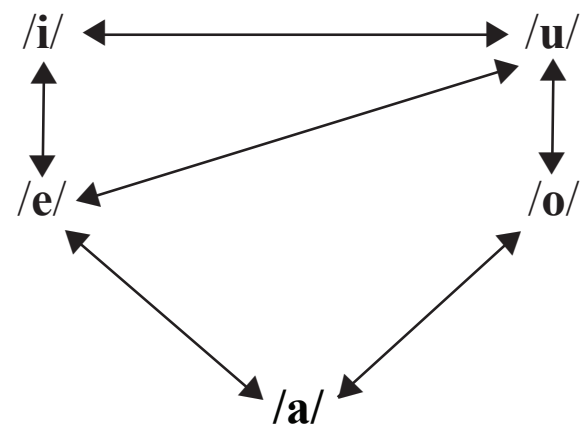

Figura 3. Variación articulatoria de vocales entre el wichí de Ingeniero Juárez y el wichí de Tartagal

Como se visualiza en la figura 3 la variación más extrema es /u/ vs. /e/, porque implica tres tipos de cambios: punto articulación (/u/ es posterior y /e/ anterior), altura (/u/ es alta y /e/ media) y redondeamiento (/u/ es redondeada y /e/ no). La variación /a/ vs. /o/ también implica cambio de punto de articulación, de altura y de redondeamiento pero, tal como lo demuestra la figura 3, la distancia en el espacio vocálico entre estas dos vocales es menor que la distancia entre las vocales $/ \mathrm{u} / \mathrm{y} / \mathrm{e} /$. Esta mayor distancia en el espacio vocálico significa que entre las vocales $/ \mathrm{u} / \mathrm{y} / \mathrm{e} / \mathrm{el}$ contraste es mayor que entre /a/ y /o/ (Flemming 2004). ${ }^{5}$

${ }^{5}$ De acuerdo con Flemming (2004) el éxito de la comunicación depende de la capacidad de los oyentes para recuperar lo que el hablante está diciendo. Por ello es importante que las categorías distintivas (como los segmentos y las palabras) no se confundan fácilmente desde un punto de vista perceptual. Dado que los sonidos vocálicos constituyen un continuo (en el sentido de que no existe un límite exacto entre un tipo de vocal y otro) la identificación de fonemas en ese continuo o espacio multi-dimensional (donde la calidad de las vocales se distinguen por los formantes o resonancias) está relacionada a su percepción y distinción. Los sonidos vocálicos más cercanos en el espacio son más similares y por lo tanto, más fáciles de confundir entre sí. En consecuencia, cuanto mayor es la distancia entre dos o más fonemas, mayor es el contraste entre ellos. 


\section{Antecedentes}

Son dos los estudios que tratan este fenómeno de variación vocálica inter-dialectal: Messineo y Braunstein (1990) y Terraza (2003). Cabe mencionar que Gerzenstein (1992) menciona y ejemplifica esta temática aunque no brinda una explicación del mismo.

\subsection{Messineo y Braunstein (1990)}

Messineo y Braunstein presentan las diferencias fonológicas, morfológicas y lexicales entre dos variedades, el wichí estudiado por Viñas Urquiza conocido como vejoz (hablado en las comunidades Misión Chaqueña y Misión la Paz de Salta) y el wichí bazanero (hablado en la comunidad Juan G. Bazán de Formosa).

Una de las diferencias fonológicas señalada por los autores es la variación vocálica que denominan corrimiento vocálico. Al respecto indican que es "un corrimiento regular condicionado por contextos específicos" pero no especifican esos condicionamientos. Dicho corrimiento es presentado por los autores en la tabla 3. Como podemos ver, es la misma variación vocálica observada entre Tartagal e Ingeniero Juárez.

Tabla 3. Corrimiento vocálico entre el wichí bazanero y vejoz (Messineo y Braunstein 1990).

\begin{tabular}{|c|c|}
\hline Bazanero & Vejoz \\
\hline$/ \mathrm{a} /$ & $/ \mathrm{e} /$ \\
\hline$/ \mathrm{e} /$ & $/ \mathrm{u} /$ \\
\hline $\mathrm{i} /$ & $/ \ddot{\mathrm{a}} / 6$ \\
\hline$/ \mathrm{o} /$ & $/ \mathrm{a} /$ \\
\hline$/ \mathrm{u} /$ & $/ \mathrm{o} /$ \\
\hline
\end{tabular}

\subsection{Terraza (2003)}

Terraza analiza y explica solamente la variación /o/ vs. /u/ entre el wichí de Rivadavia (sur de Salta) y el de Tartagal. Según la autora, esta variación se debe a que la realización fonética del fonema /o/ en Rivadavia es semi-abierta [0], mientras que en Tartagal es semi-cerrada [o]; por lo tanto, cuando en Tartagal se encuentra /o/, en Rivadavia se encuentra /u/.

${ }^{6}$ El símbolo /ä/ representa a una vocal baja y posterior. Como mencionamos al inicio de este capítulo, algunos autores, como en este caso, identifican el fonema $/ \mathrm{a} /$. 
CAYRÉ BAITO - UNA PRIMERA APROXIMACIÓN A LA VARIACIÓN VOCÁLICA ...

\section{Breve descripción fonética de las vocales de Tartagal e Ingeniero Juárez}

En las tablas 4.1 y 4.2 presentamos los valores de los formantes F1 y F2 de las cinco vocales $^{7}$ para apreciar mejor las similitudes y diferencias.

Tabla 4.1. Valores (media) de los formantes F1 y F2 en Hz de las vocales en Tartagal.

\begin{tabular}{|c|c|c|c|}
\hline \multicolumn{2}{|c|}{ Vocales } & F1 (media) & F2 (media) \\
\hline \multirow{2}{*}{ Altas } & $/ \mathrm{i} /$ & 392.43 & 2102.28 \\
\cline { 2 - 4 } & $/ \mathrm{u} /$ & 412.3 & 1053.17 \\
\hline \multirow{2}{*}{ Medias } & $/ \mathrm{e} /$ & 500.31 & 1826.86 \\
\cline { 2 - 4 } & $/ \mathrm{o} /$ & 532.07 & 997.28 \\
\hline Bajas & $/ \mathrm{a} /$ & 614.2 & 1295.47 \\
\hline
\end{tabular}

Tabla 4.2. Valores (media) de los formantes F1 y F2 en Hz de las vocales en Ingeniero Juárez.

\begin{tabular}{|c|c|c|c|}
\hline \multicolumn{2}{|c|}{ Vocales } & F1 (media) & F2 (media) \\
\hline \multirow{2}{*}{ Altas } & $/ \mathrm{i} /$ & 333.80 & 2148.61 \\
\cline { 2 - 4 } & $/ \mathrm{u} /$ & 386.50 & 989.87 \\
\hline \multirow{2}{*}{ Medias } & $/ \mathrm{e} /$ & 510.54 & 1805.9 \\
\cline { 2 - 4 } & $/ \mathrm{o} /$ & 520.52 & 1047.01 \\
\hline Bajas & $/ \mathrm{a} /$ & 635.15 & 1222.64 \\
\hline
\end{tabular}

En base a estos datos podemos ver que en ambas variedades la vocal baja /a/ se realiza como central [e]; las vocales anteriores /i/ y /e/ y la vocal posterior /u/ son articulatoriamente más abiertas y se realizan fonéticamente como $[\mathrm{I}],[\varepsilon]$ y $[\mho]$. Pero existen entre ambas variedades algunas diferencias que podrían brindar datos de interés para el análisis de las variaciones /o/ vs. /u/ y /u/ vs. /e/.

Por un lado, la calidad vocálica de /o/ en Tartagal es articulatoriamente más posterior y se realiza como [o], mientras que en Ingeniero Juárez es más anterior y se realiza como [э]. Esta diferencia con respecto a la posterioridad de la vocal /o/ permite explicar, tal como señala Terraza (2003), la variación /o/ vs. /u/.

Por otro lado, la vocal /u/ presenta un F1 con valores más altos en Tartagal que en Ingeniero Juárez. El valor media del F1 de /u/ en Tartagal es de 412Hz, mientras que en Ingeniero Juárez tiene un valor de menor frecuencia, $386 \mathrm{~Hz}$. Esta diferencia con respecto a la altura de la vocal /u/ podría explicar la variación /u/ vs. /e/: como la vocal /u/ de Tartagal tiene valores de F1 relativamente altos, se aproxima a los valores de la vocal media /e/. Sin embargo, observamos que en los dos sistemas fonológicos hay una tendencia de la vocal /u/ hacia la vocal /e/. Podemos visualizar esta tendencia en las figuras 4.1 y 4.2 que muestran los valores de F1 y F2 de las vocales y su dispersión en el espacio. El eje de las ordenadas indica los valores de F1 y el eje de las abscisas, los valores de F2.

${ }^{7}$ Para poder determinar la calidad fonética de las vocales hemos medido la frecuencia de los formantes más bajos, F1 (para ver altura) y F2 (para ver punto de articulación). Los formantes fueron medidos manualmente utilizando Praat. Cada muestra de las vocales (20 muestras para cada vocal) fue medida segmentando un intervalo corto de tiempo (entre 30 y 50ms) durante la parte más estable de la vocal, cerca del medio de la misma. 


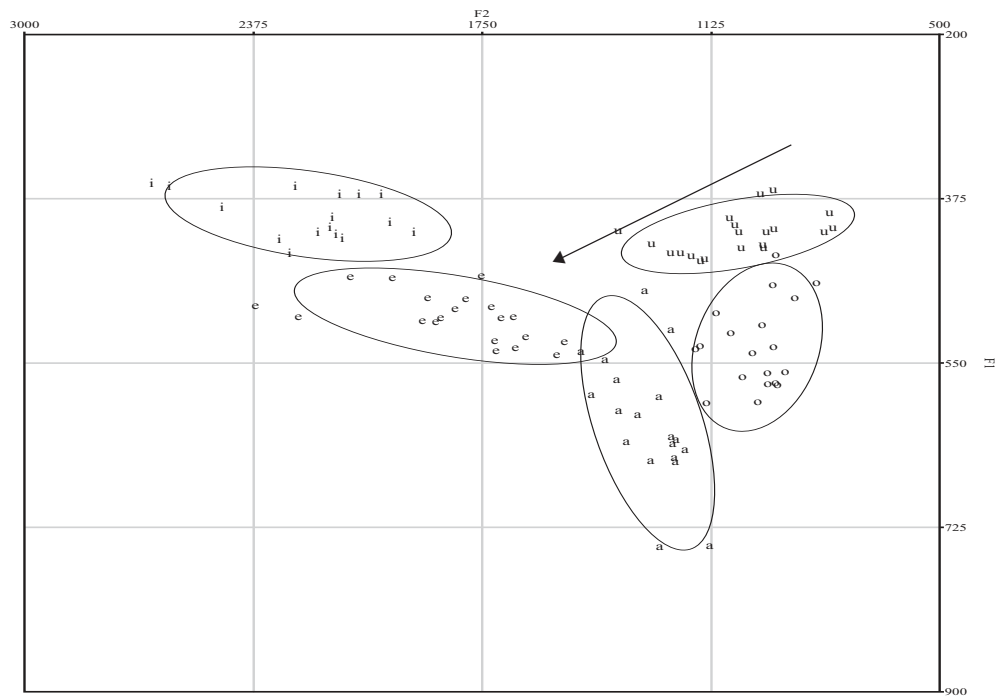

Figura 4.1. Diagrama de dispersión de las vocales en Tartagal (basado en el idiolecto de JB). La flecha indica la tendencia de la vocal/u/hacia la vocal /e/.

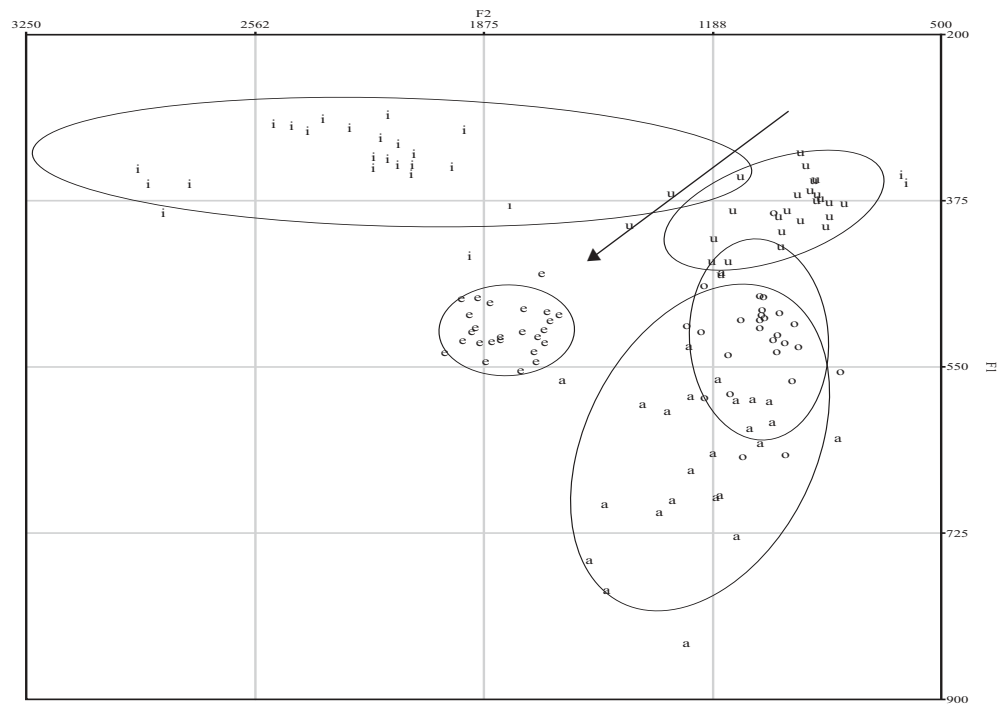

Figura 4.2. Diagrama de dispersión de las vocales en Ingeniero Juárez (basado en el idiolecto de SM). La flecha indica la tendencia de la vocal/u/ hacia la vocal /e/.

\section{Una primera explicación diacrónica para la variación vocálica inter-dialectal}

De acuerdo con Najlis (1984) el proto-mataguayo tenía un sistema de 8 vocales. Según la autora, la dificultad de mantener el contraste entre estas vocales generó cambios en cadena que redujeron el sistema vocálico a seis unidades como se observa en la figura 5. 
CAYRÉ BAITO - UNA PRIMERA APROXIMACIÓN A LA VARIACIÓN VOCÁLICA ...

Tabla5. Sistema vocálico del

proto-mataguayo (Najlis, 1984: 15) ${ }^{8}$
Figura 5. Sistema de vocales según

Najlis (1984: 44).

\begin{tabular}{|c|c|c|}
\hline & Anterior & Posterior \\
\hline Alta & i & $\mathrm{u}$ \\
\hline Semi-cerrada & $\mathrm{E}$ & $\mathrm{O}$ \\
\hline Semi-abierta & $\mathrm{e}$ & $\mathrm{o}$ \\
\hline Baja & $\mathrm{a}$ & $\mathrm{A}$ \\
\hline
\end{tabular}

$\begin{array}{lll}\mathrm{i} & \mathrm{H} & \mathrm{u} \\ \mathrm{e} & & \mathrm{o}\end{array}$

a

No estamos seguros a qué símbolo del IPA corresponde el símbolo $t$ usado por Najlis, probablemente sea la vocal posterior no redondeada $u$. Respecto a $u$, Najlis sólo menciona que es el alófono no redondeado de la vocal posterior $u$, que luego se convierte en fonema, pero en los sistemas vocálicos actuales del wichí no existe un fonema posterior alto no redondeado.

Es posible resumir los cambios mencionados por Najlis en dos principales. Un cambio que afectó a las proto-vocales $O, E$ y $A$ y otro, que afectó a las proto-vocales $a$, $e$ y $u$. Obsérvese que estos cambios tuvieron en todos los casos, dos direcciones posibles (por lo tanto dos resultados posibles) generando así, la variación dialectal observada en la actualidad. Respecto a la vocal media abierta $o$ y la vocal anterior alta $i$ Najlis indica que no sufrieron ningún cambio.

1. Cambio que afectó a las vocales $O, E$ y $A$ reduciendo el sistema de ocho vocales a cinco.

- La vocal media cerrada $O$ cambia a $u$ en el wichí del sur ${ }^{9}$ y a $o$ en los otros dialectos. Las letras T e IJ hacen referencia a Tartagal e Ingeniero Juárez respectivamente.

Tabla 6.1. Ejemplos del cambio que afectó a la vocal $O$.

\begin{tabular}{|c|c|c|c|c|}
\hline \multirow{2}{*}{$\begin{array}{c}\text { Cambio } \\
\text { vocálico }\end{array}$} & Proto-mataguayo & $\begin{array}{c}\text { Wichí (datos de Najlis) } \\
\text {-las barras separan ejemplos de } \\
\text { las distintas variedades- }\end{array}$ & Wichí (T) & Wichí (IJ) \\
\hline \multirow{3}{*}{$\mathrm{O}>\mathrm{o}, \mathrm{u}$} & $\mathrm{wO}$ 'cuello' & $\mathrm{wo} / \mathrm{wu}$ & $\mathrm{wo}$ & wu \\
\cline { 2 - 6 } & $\mathrm{h}^{\mathrm{w}}$ ajhnO 'carbón' & $\mathrm{h}^{\mathrm{w}}$ aihnjo / hwaihnju & $\mathrm{x}^{\mathrm{w}} \mathrm{i}$ 'jo & $\mathrm{x}^{\mathrm{w}} \mathrm{i}$ 'ju \\
\cline { 2 - 6 } & sOnatha 'oveja' & sonata / sunatax & tsona'tax & tsuna'tax \\
\cline { 2 - 6 } & hnolO 'arena' & xõlu / xolo & ho'lo & hu'lu \\
\hline
\end{tabular}

- La vocal media cerrada $E$ cambia a $i$ en algunos dialectos y a $e$ en otros.

${ }^{8}$ Creemos que los símbolos usados por Najlis A, a, E, e, O y o se corresponden con los siguientes símbolos del IPA a, a, e, $\varepsilon, o$, o.

${ }^{9}$ Los datos de Najlis son de tres variedades, una de Misión La Paz, Salta; otra de Pozo Yacaré, Formosa y la última de Algarrobal, Salta. Najlis se refiere a estas variedades como variedades del norte, del sur y del oeste respectivamente. De acuerdo a esta distinción dialectal, la variedad de Tartagal correspondería a la variedad del norte y la de Ingeniero Juárez a la del sur. 
Tabla 6.2. Ejemplos del cambio que afectó a la vocal $E$.

\begin{tabular}{|c|c|c|c|c|}
\hline Cambio vocálico & Proto-mataguayo & $\begin{array}{c}\text { Wichí (datos de } \\
\text { Najlis) } \\
\text {-las barras separan } \\
\text { ejemplos de las } \\
\text { distintas variedades- }\end{array}$ & Wichí (T) & Wichí (IJ) \\
\hline \multirow{4}{*}{$E>i, e$} & hwEhlE 'sueño' & hwehlek/hwihlek & $\mathrm{x}^{\mathrm{w}} \mathbf{i}$ 'łan 'soñar' & $\mathrm{x}^{\mathrm{w}} \mathbf{e}^{\prime} \mathrm{tan}$ \\
\hline & najEhn 'camino' & Najex & na'jix & no'jix \\
\hline & nEjAwk 'soga' & nijAwk/nijakw/nijok & ni'jak $^{w}$ & ni'jok ${ }^{w}$ 'hilo' \\
\hline & acEhna 'mujer' & ačihna / acihna & tsi'na & atsi'na \\
\hline
\end{tabular}

- La vocal baja posterior $A$ cambia a $o$ en los dialectos del sur pero a $a$ en los otros. En algunas palabras, como mostramos en la tabla 6.4, $A$ cambia a $o$ ante oclusiva velar o ante w. En nuestros datos $A$ cambia a $o$ en Tartagal pero a $u$ en Ingeniero Juárez.

Tabla 6.3. Ejemplos del cambio que afectó a la vocal $A$.

\begin{tabular}{|c|c|c|c|c|}
\hline $\begin{array}{c}\text { Cambio } \\
\text { vocálico }\end{array}$ & Proto-mataguayo & $\begin{array}{c}\text { Wichí (datos de Najlis) } \\
\text {-las barras separan ejemplos } \\
\text { de las distintas variedades- }\end{array}$ & Wichí (T) & Wichí (IJ) \\
\hline \multirow{3}{*}{ A $>$ o, a } & mA 'dormir' & ma & ma & mo \\
\cline { 2 - 5 } & ihnAt 'agua' & inot / inat & i'nat & i'not \\
\cline { 2 - 5 } & pA-ahn-Ajn 'pimiento' & paahnan / poahnu & pa'nnan & po'nnon \\
\cline { 2 - 5 } & t'Ahn 'piel' & t'ax / t'ox & t'ax 'cuero' & t?ox 'cuero' \\
\hline
\end{tabular}

Tabla 6.4. Ejemplos del cambio que afectó a la vocal $A$ por contexto fonético.

\begin{tabular}{|c|c|c|c|c|}
\hline $\begin{array}{c}\text { Cambio } \\
\text { vocálico }\end{array}$ & Proto-mataguayo & $\begin{array}{c}\text { Wichí (datos de Najlis) } \\
\text {-las barras separan ejemplos } \\
\text { de las distintas variedades- }\end{array}$ & Wichí (T) & Wichí (IJ) \\
\hline A> o, u & hmAk 'polvo' & mok & mok $^{\mathrm{w}}$ & muk $^{\mathrm{w}}$ \\
\hline & $\begin{array}{c}\text { tAwehn 'agujero } \\
\text { del recipiente' }\end{array}$ & towex & to'wex 'olla' & tu'wex 'olla' \\
\hline
\end{tabular}

En la figura 6 esquematizamos estos cambios, entre paréntesis colocamos los símbolos usados por Najlis.

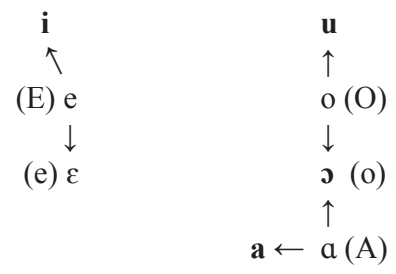

Figura 6. Cambio que redujo el proto-sistema de 8 vocales a 5 . 
Como podemos observar en la figura 6, el cambio que afectó a las proto-vocales $O$, $E$ y $A$ tiene dos direcciones posibles; por un lado, cambio de vocal [+ATR] a [-ATR]; por otro, cambio de altura creciente: de vocal media a alta o de vocal baja a media.

Cambio de vocal [+ATR] a [-ATR] Cambio de altura creciente
(E) $/ \mathrm{e} / \rightarrow / \varepsilon /$
$(\mathrm{E}) / \mathrm{e} / \quad \rightarrow / \mathrm{i} /$
(O) $/ \mathrm{o} / \mathrm{a} \rightarrow \mathrm{d} /$
(O) $/ \mathrm{o} / \mathrm{h} \rightarrow \mathrm{u} /$
(A) $/ \mathbf{a} / \quad \rightarrow / \mathbf{a} /$
(A) $/ \mathrm{a} / \mathrm{l} / \mathrm{o}$

La variedad de Tartagal siguió la primera dirección mientras que la variedad de Ingeniero Juárez siguió la segunda pero ambas direcciones del cambio apuntaron a eliminar la oposición entre vocales [+ATR] y [-ATR] y priorizar las vocales [-ATR], como podemos visualizar en las figuras 7.1 y 7.2 .

\section{Sistema fonológico de T}
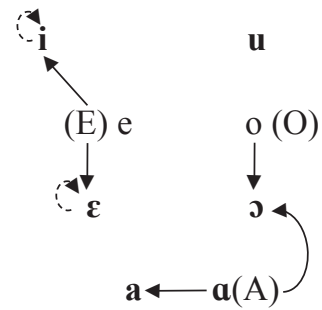

Figura 7.1. Variación vocálica en Tartagal (T).

\section{Sistema fonológico de IJ}
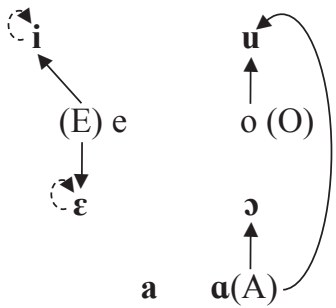

Figura 7.2. Variación vocálica en Ingeniero Juárez (IJ).

2. Cambio que afectó a las vocales $a, e$ y $u$.

- La cercanía del punto de articulación entre $a$ y la vocal media abierta $e$ produjo cambios irregulares en dos sentidos:

a $>$ e (en algunas palabras cuando $a$ está precedida de una consonante oclusiva velar final).

$>\mathrm{e}>\mathrm{a}$ (en algunas palabras cuando $e$ está precedida de una consonante palatal pero con diferencias entre las variedades del norte y del sur).

Tabla 7. Ejemplos de los cambios a $>$ e y e $>$ a.

\begin{tabular}{|c|c|c|c|c|}
\hline \multirow{2}{*}{ Cambiovocálico } & Proto-mataguayo & $\begin{array}{c}\text { Wichí (dato de } \\
\text { Najlis) }\end{array}$ & $\begin{array}{c}\text { Wichí } \\
\text { (T) }\end{array}$ & $\begin{array}{c}\text { Wichí } \\
\text { (IJ) }\end{array}$ \\
\hline \multirow{2}{*}{$\begin{array}{c}\text { a> e } \\
\text { e a }\end{array}$} & sakAl 'alma' & hnusek/hnosek & hu'sek & hu'sek \\
\cline { 2 - 5 } & jewu 'chamán' & xajawu & haja'wu & haja'we \\
\cline { 2 - 5 } & çehl(h)nO ‘quirquincho' & çahno (S) çehno (N) & tce'no & tJa'no \\
\hline
\end{tabular}


- Respecto a la vocal $u$ Najlis sólo menciona que en las variedades del sur se produce un alófono no redondeado, en casi todas las posiciones, que luego se convierte en fonema. El rasgo redondeado de $u$ se mantiene cuando está seguida de una consonante continua labial. ${ }^{10}$ Dado que no es un fonema en los sistemas vocálicos actuales del wichí podemos asumir que fue fonema en un estado previo.

Si comparamos nuestros datos con los ejemplos del proto-mataguayo de Najlis se puede observar que las proto-vocales $a$ y $o$ se mantienen en Tartagal pero cambian a $o$ у $u$ respectivamente, en Ingeniero Juárez. De igual amanera, la proto-vocal $u$ se mantiene en Tartagal pero cambian a $i$ y $e$ en Ingeniero Juárez. La figura 8 muestra estos cambios, entre paréntesis colocamos los símbolos usados por Najlis.

Tabla 8. Ejemplos de los cambios a $>0, o>$, u $>$ e y $u>i$.

\begin{tabular}{|c|c|c|c|c|}
\hline Cambio vocálico & Proto-mataguayo & Wichí (datos de Najlis) & Wichí ( T) & Wichí (IJ) \\
\hline \multirow[t]{2}{*}{$a>0$} & najEhn 'camino' & najex & na'jix & no'jix \\
\hline & qala 'pierna' & kala / kala & ka'la & ko'lo \\
\hline \multirow[t]{2}{*}{$\mathrm{o}>\mathrm{u}$} & ihno 'hombre' & xino? & hi'no & hi'nu \\
\hline & $\begin{array}{l}\text { mok'o wok'o } \\
\text { 'búho' }\end{array}$ & wok'o & wo'k ${ }^{?} \mathbf{o}$ & wu'k $\mathbf{u}^{3} \mathbf{u}$ \\
\hline \multirow[t]{2}{*}{$\mathrm{u}>\mathrm{e}$} & nap'u 'lamer' & nap'u & na'p $p^{2} \mathbf{u}$ & na'p? \\
\hline & pulE 'cielo' & pule / pule & pu'le & pe'le \\
\hline $\mathrm{u}>\mathrm{i}$ & hlhnup 'nido' & hlup / hlüp & tup & tip \\
\hline
\end{tabular}

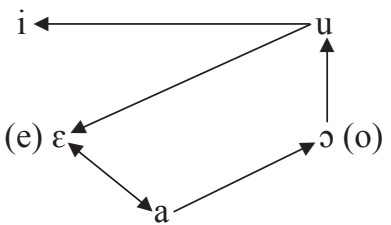

Figura 8. Cambio que afectó a las proto-vocales $a, o$ у $u$.

Para poder ver la frecuencia con que ocurren las variaciones entre Tartagal e Ingeniero Juárez hemos comparado un total de 229 palabras y hemos observado lo siguiente:

1. El $63 \%$ de las palabras presenta variación y el 37\% no presenta variación esto significa que en ambos sistemas fonológicos se mantienen las mismas vocales, por ejemplo: $i h i$ 'estar' en Tartagal es también ihi en Ingeniero Juárez.

${ }^{10}$ A continuación trascribimos el ejemplo mostrado por Najlis para explicar este cambio, MA significa 'mataco':

tucha 'humo' MA (dialecto del sur) tucax MA (otros dialectos) tucax

jewu 'chamán’ MA xajawu 
CAYRÉ BAITO - UNA PRIMERA APROXIMACIÓN A LA VARIACIÓN VOCÁLICA ...

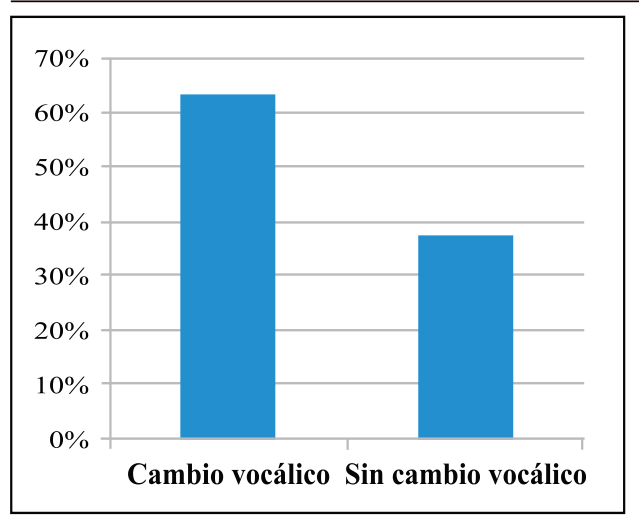

\begin{tabular}{|c|c|c|}
\hline & Cantidad & Porcentaje \\
\hline Cambio vocálico & 145 & $63 \%$ \\
\hline $\begin{array}{c}\text { Sin cambio } \\
\text { vocálico }\end{array}$ & 84 & $37 \%$ \\
\hline Total & 229 & $100 \%$ \\
\hline
\end{tabular}

Gráfico 1. Porcentaje de palabras que presenta cambio vocálico y que no presentan cambio vocálico

2. Las variaciones más frecuentes son /a/ vs. /o/, /o/ vs. /u/ y /e/ vs. /u/. La frecuencia de /a/ vs. /e/, /i/ vs. /e/ y /u/ vs. /i/ es menos del $6 \%$.

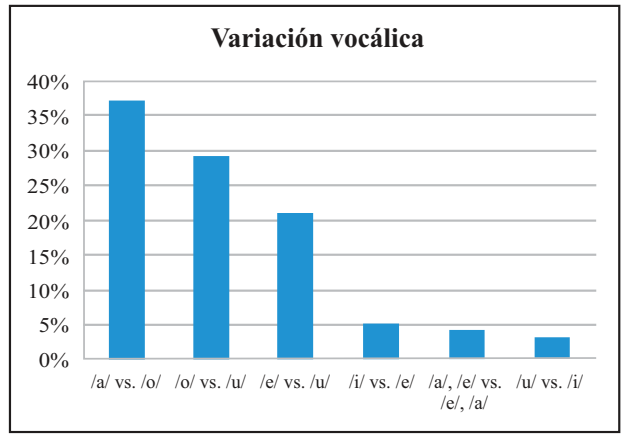

\begin{tabular}{|l|c|c|}
\hline Variación vocálica & Cantidad & Porcentaje \\
\hline /a/ vs. /o/ & 55 & $37 \%$ \\
\hline /o/ vs. /u/ & 44 & $29 \%$ \\
\hline /e/ vs. /u/ & 32 & $21 \%$ \\
\hline /i/ vs. /e/ & 7 & $5 \%$ \\
\hline $\begin{array}{l}\text { /a/ vs. /e/ } \\
\text { /e/ vs. /a/ }\end{array}$ & 6 & $4 \%$ \\
\hline /u/ vs. /i/ & 5 & $3 \%$ \\
\hline Total & 149 & $100 \%$ \\
\hline
\end{tabular}

Gráfico 2. Frecuencia de aparición de las variaciones vocálicas

3. Del grupo de palabras que no presenta variación, las vocales posteriores casi no se mantienen en ambas variedades, el número de ejemplos registrado es menor al $5 \%$.

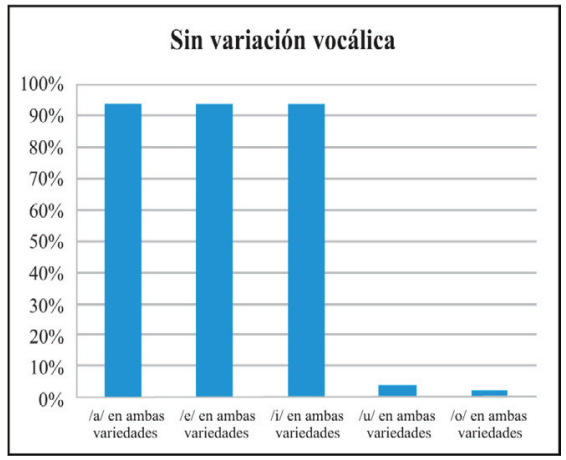

\begin{tabular}{|c|c|c|}
\hline \multicolumn{2}{|c|}{ Sin variación vocálica } \\
\hline \multirow{2}{*}{ Vocal presente } & Cantidad & Porcentaje \\
& 84 & $100 \%$ \\
\hline /a/ en ambas variedades & \multicolumn{2}{|c|}{} \\
\cline { 1 - 1 } /e/ en ambas variedades & \multirow{2}{*}{$94 \%$} \\
\hline /i/ en ambas variedades & \\
\hline /u/ en ambas variedades & \multicolumn{2}{|c|}{$4 \%$} \\
\hline /o/ en ambas variedades & $2 \%$ \\
\hline
\end{tabular}

Gráfico 3. Frecuencia de aparición de las vocales que pueden estar presentes en ambas variedades. 
En base a los datos presentados previamente, podemos decir que el sistema fonológico de Tartagal es más conservador con respecto a la proto-lengua que el sistema fonológico de Ingeniero Juárez.

\section{Sistema fonológico de $T$}

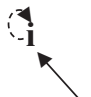

(E)
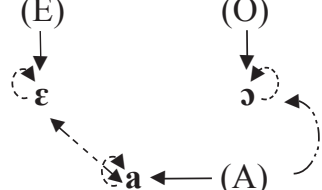

Figura 9. 1. Variación vocálica en Tartagal (T).

\section{Sistema fonológico de IJ}

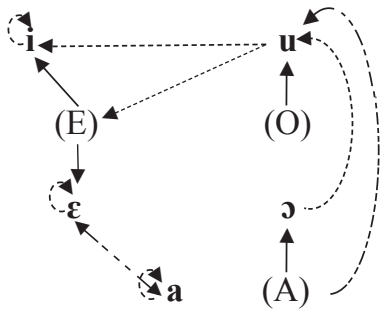

Figura 9. 2. Variación vocálica en Ingeniero Juárez (IJ)

\section{Referencias:}

$\longrightarrow$ Cambio que afecta a las proto-vocales $A, E$ and $\mathrm{O}$ (en los dos sistemas).Véase tablas $6.1,6.2$ y 6.3

Cambio que afecta a las proto-vocales $a$ y $A$ debido al contexto fonético (en los dos sistemas).

Véase tablas 6.4 y 7

Proto-vocales que se mantienen en los sistemas

$\rightarrow$ Cambio que afecta a las proto-vocales $a, o$ y $u$ (sólo en IJ). Véase tabla 8.

Si la variación es menos frecuente entre las vocales anteriores es posible plantear como hipótesis que la variación vocálica entre los dos sistemas es probablemente el resultado de un cambio con cierta orientación que va de la vocal central /a/ y de las vocales posteriores $/ \mathrm{o} / \mathrm{y} / \mathrm{u} /$ hacia las anteriores (mantenidas en gran número de palabras en las dos variedades según el gráfico 3). Esta orientación se combina a su vez, con un cambio de altura creciente. En la figura 10 representamos esta posible dirección del cambio.

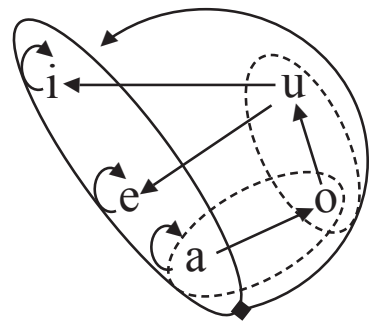

Figura 10. Posible dirección del cambio vocálico. 
Nada de variación o menos variación

Variación

Dirección del cambio

La dirección del cambio que proponemos en este análisis se corresponde con uno de los patrones de cambio propuestos por Labov (1994) que combina dos de los principios que regulan los cambios en cadena: 1) las vocales posteriores se mueven hacia las anteriores y 2) las vocales largas suben en altura. En (1a) presentamos el esquema de Labov (1994: 129-131) para ilustrar este patrón y en (1b), uno de los cambios vocálicos presentados por el autor para ejemplificarlo. Los datos presentados en (1b) corresponden al albanés. ${ }^{11}$

(1)

(a)

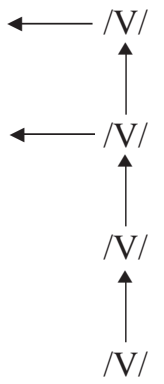

(b)

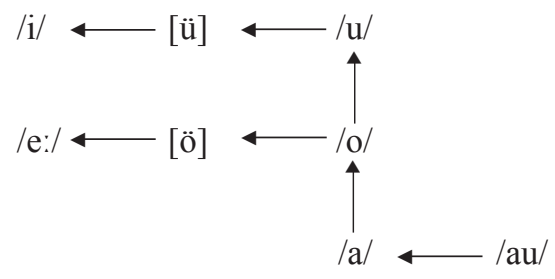

En (2) podemos ver que es posible aplicar este patrón a los datos de variación que observamos en wichí, aunque las vocales que incrementan en altura no son largas (véase figuras 9.1 y 9.2).

(2)

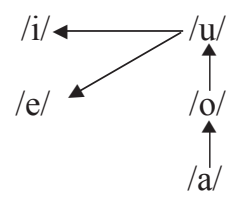

La excepción al patrón propuesto por Labov sería el cambio de la vocal alta /u/ a la vocal media /e/, puesto que la altura baja en vez de incrementar. Creemos que esta excepción puede explicarse como resultado del siguiente cambio diacrónico: /u/ $>/ \mathrm{u} /$ (pérdida del rasgo redondeado) ${ }^{12}>\mathrm{i}>\mathrm{e}$. Esta hipótesis puede confirmarse por los siguientes hechos:

${ }^{11}$ De acuerdo con Labov (1994: 131) las vocales $\overline{\mathbf{a}}, \overline{\mathbf{o}}$ y $\overline{\mathbf{u}}$ el diptongo au del protoindo-europeo están representados en albanés por /o/, /e/, /i/ y /a/, respectivamente.

${ }^{12}$ Recordemos que con respecto a la proto-vocal $u$ Najlis menciona que en las variedades del sur se produce un alófono no redondeado $t$, en casi todas las posiciones, que luego se convierte en fonema. 

/u/ vs. /i/.

- Sólo 2\% de las palabras comparadas entre ambos sistemas presenta la variación

- Sólo 5\% de las palabras comparadas presenta la variación /e/ vs. /i/ (resultado del cambio $\mathrm{E}>\mathrm{e}, \mathrm{i})$.

- Sólo hemos registrado un ejemplo donde la proto-vocal $i$ se corresponde con $e$ en el sistema de Ingeniero Juárez: *hmi 'amar' > hu'min (T) vs. he'men (IJ).

Partimos de la hipótesis que este cambio diacrónico es consecuencia de dos tendencias que han regulado los cambios vocálicos en wichí, por un lado, el movimiento de vocales posteriores hacia las anteriores; por otro, la preferencia por las vocales [-ATR]. Esta preferencia se manifiesta actualmente en los sistemas fonológicos de Tartagal e Ingeniero Juárez puesto que las vocales anteriores /i/ y /e/ y la vocal posterior $/ \mathrm{u} /$ son articulatoriamente más abiertas y se realizan fonéticamente como $[\mathrm{I}],[\varepsilon]$ y $[\mho]$. Si esta hipótesis es correcta, podríamos explicar la tendencia actual de la vocal / $\mathrm{u} / \mathrm{hacia}$ la vocal /e/ que observamos en ambos sistemas fonológicos (ver figuras 4.1 y 4.2). La vocal $/ \mathrm{u} /$ presenta en ambos sistemas fonológicos un F1 con valores que superan los $350 \mathrm{~Hz}$ y se aproximan a los valores de una vocal media (ver sección 4.1). Dado que la orientación del cambio, tal como indicamos en la figura 10, va de las vocales posteriores a las anteriores entonces la vocal anterior media más próxima a los valores de F1 de /u/ sería /e/.

\section{La variación vocálica en otras variedades del wichí}

Comparando nuestros datos con estudios fonológicos de diferentes variedades del wichí, podemos hipotetizar que esta variación fonológica distingue dos grandes variedades dialectales. Por un lado, el wichí hablado en Bolivia (Claesson 1994) y el norte de Salta (Viñas Urquiza 1976; Fernández Garay y Spinelli 2009 y Avram 2008), que sería más conservador como Tartagal. Por otro lado, el wichí hablado en el sur de Salta (Terraza 2009), Formosa y Chaco (Censabella 2009b y Nercesian 2011), que sería menos conservador como Ingeniero Juárez. En la tabla 9 brindamos algunos ejemplos que muestran la variación fonológica entre estas dos grandes variedades. Los ejemplos han sido transcriptos tal como están en las fuentes; entre paréntesis colocamos la sigla de la variedad a la que corresponde cada ejemplo: V 'vejoz' (figura $2 \mathrm{~N}^{\circ} 9$ ), $\mathrm{N}$ 'nocten' (Bolivia), RN 'Rivadavia Banda Norte' (figura 2, $\mathrm{N}^{\circ} 2$ y 3), MLP 'Misión la Paz' (figura 2, $\mathrm{N}^{\circ} 2$ ), $\mathrm{T}$ 'Tartagal' (figura 2, $\mathrm{N}^{\circ} 2$ ), RS 'Rivadavia Banda Sur' (figura 2, $\mathrm{N}^{\circ}$ 14), F-CH 'Formosa y Chaco' (figura $2 \mathrm{~N}^{\circ} 18,16$ y 20) e IJ 'Ingeniero Juárez' (figura $\left.2, \mathrm{~N}^{\circ} 16\right)$. 
CAYRÉ BAITO - UNA PRIMERA APROXIMACIÓN A LA VARIACIÓN VOCÁLICA ...

Tabla 9. Variación vocálica entre variedades wichí habladas en Bolivia y norte de Salta y variedades habladas en el sur de Salta, Chaco y Formosa.

\begin{tabular}{|c|c|c|}
\hline Variación & Bolivia y Norte de Salta & Sur de Salta, Formosa y Chaco \\
\hline /a/ vs. /o/ & $\begin{array}{l}\text { niisàhes 'zapatos' (N) } \\
\text { haPla 'árbol' (V) } \\
\text { inat 'agua' (MLP) } \\
\text { [na'yix] 'camino' (RN) }\end{array}$ & $\begin{array}{l}\text { nisohes 'zapatos' (RS) } \\
\text { halo ‘árbol' (IJ) } \\
\text { Pinot 'camino' (F-CH) } \\
\text { no'yix 'camino' (RS) }\end{array}$ \\
\hline /o/ vs. /u/ & $\begin{array}{l}\text { Pooqoh 'mi mamá' (N) } \\
\text { [čokok] 'mariposa' (RN) } \\
\text { kºhõt 'araña' (MLP) } \\
\text { mok ' }^{\text {? }} \text { 'polvo' (V) }\end{array}$ & 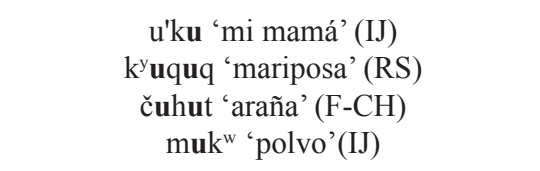 \\
\hline /u/ vs. /e/ & 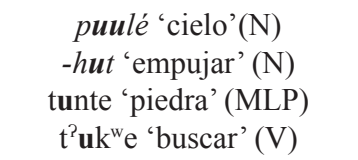 & $\begin{array}{l}\text { pele 'cielo' (RS) } \\
\text {-het 'empujar' (IJ) } \\
\text { tente 'piedra' (F-CH) } \\
\text { t'ek e'e 'él busca' (RS) }\end{array}$ \\
\hline /e/ vs. /a/ & 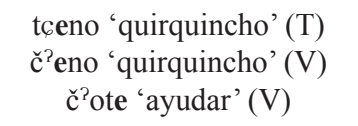 & $\begin{array}{c}\mathrm{k}^{\mathrm{y}} \mathbf{a n h u} \text { 'quirquincho' }(\mathrm{R}) \\
\text { [ča'nu] 'quirquincho' (CH-F) } \\
\mathrm{k}^{\mathrm{j}} \mathrm{uta}(\mathrm{RS})\end{array}$ \\
\hline /i/ vs. /e/ & $\begin{array}{c}\text { yahuuminh 'le gusta' (N) } \\
\text { Pnõhũmin 'amante' (MLP) } \\
\text { hi'lu 'yica, bolsa' (V) } \\
\text { li'čc 'u 'huevo' (V) }\end{array}$ & $\begin{array}{l}\text { nhemen 'quiero' (RS) } \\
\text { ujhemen ‘quiero' (IJ) } \\
\text { he'le ‘yica' (RS) } \\
\text { te'k'e 'huevo' (RS) }\end{array}$ \\
\hline $\begin{array}{l}\text { /u/ vs. /i/, } \\
/ \mathrm{e} /\end{array}$ & 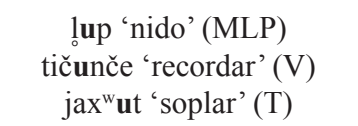 & 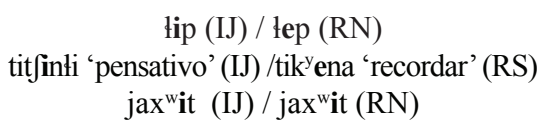 \\
\hline
\end{tabular}

Fernández Garay y Spinelli (2009) han observado las siguientes alternancias: e $\sim$ i; $\mathrm{e} \sim \mathrm{u} ; \mathrm{o} \sim \mathrm{a} ; \mathrm{o} \sim \mathrm{u} ; \mathrm{o} \sim \Lambda$ en hablantes de la misma comunidad o en hablantes de distintas comunidades del norte de Salta.

\section{Conclusión}

La variación fonológica de vocales entre las variedades del wichí es uno de los aspectos más interesantes que presenta la compleja continuidad dialectal que caracteriza a esta lengua. Los estudios previos que existen en torno a esta problemática son pocos (Messineo y Braunstein 1990, Terraza 2009) y ofrecen explicaciones parciales sobre este fenómeno. Con la finalidad de mostrar una primera aproximación a este fenómeno, en este trabajo estudiamos la variación vocálica observada entre el wichí hablado en Tartagal (Salta) y en Ingeniero Juárez (Formosa) y brindamos una posible explicación diacrónica. La misma fue elaborada comparando nuestros datos con la proto-lengua y considerando las particularidades fonéticas actuales de las vocales en las variedades estudiadas. 
La comparación de nuestros datos con la proto-lengua mataguaya nos permitió demostrar que el wichí hablado en Tartagal es más conservador con respecto a la protolengua, que el wichí de Ingeniero Juárez (véase sección 5). En base a este análisis sostenemos en concordancia con Labov (1994), que la variación es resultado de un cambio histórico, regulado por dos principios: cambio de vocales posteriores hacia las anteriores y cambio de altura creciente (véase figura 10). La excepción al patrón propuesto por Labov es la variación $u$ vs $e$ puesto que la altura baja en vez de incrementar. Creemos que esta variación es resultado del siguiente cambio: $/ \mathrm{u} />/ \mathrm{u} /$ (pérdida del rasgo redondeado) $>\mathrm{i}>$ e. Partimos de la hipótesis que dicho cambio es consecuencia de dos tendencias que han regulado los cambios vocálicos en wichí, por un lado, el movimiento de vocales posteriores hacia las anteriores y por otro, la preferencia por las vocales [-ATR]. Esta preferencia se manifiesta actualmente en los sistemas fonológicos de Tartagal e Ingeniero

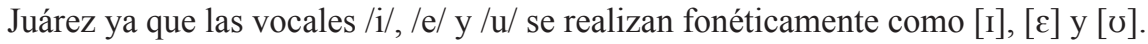

Finalmente, comparamos nuestros corpus con datos de otras variedades y observamos que la misma variación se observa entre el wichí hablado en Bolivia y norte de Salta y el wichí hablado en el sur de Salta, Chaco y Formosa (véase tabla 9). Este dato podría permitirnos bosquejar dos grandes variedades dialectales de esta lengua indígena, una del norte (más conservadora con respecto a la proto-lengua) y otra del sur.

Esta temática amerita un estudio dialectológico que permita no sólo profundizar y corroborar la dirección del cambio vocálico propuesta en este trabajo, sino también abordar interrogantes de análisis como: ¿la variación entre las dos grandes variedades dialectales (wichí del norte y wichí del sur) es estable o se observan alternancias?; ¿las alternancias registradas por Fernández Garay y Spinelli (2009) en el wichí del norte de Salta se observan también en las variedades del sur de Salta, Chaco y Formosa?; ¿por qué las variedades del norte son más conservadoras que las del sur?; ¿cómo se manifiesta esta variación vocálica en el habla de las jóvenes generaciones (niños y adolescentes)?

\section{$\overline{\text { Referencias bibliográficas }}$}

Braunstein, José (2009). Matako-Dialecto bazanero (1989): Contribución para el Intercontinental Dictionary Series Worldlist editado por Mary Ritchie Key (Universidad de California en Irvine). En José Braunstein; Cristina Messineo (comps.). Hacia una nueva carta étnica del gran Chaco VIII, pp. 3-91. Buenos Aires: Centro del Hombre Antiguo Chaqueño.

Braunstein, José; Miller, Elmer (1999). Etnohistorical introduction. En Elmer Miller (ed.). Peoples of the Gran Chaco, pp 1-22.Connecticut/Londres: Bergin y Garvey.

Censabella, Marisa Inés; Giménez Mariana; Gómez, Martín (2011). Políticas lingüísticas recientes en la provincia del Chaco (Argentina) y su posible impacto en la revitalización de las lenguas indígenas. En Marleen Haboud; Nicholas Ostler (eds.). Voces e imágenes de las lenguas en peligro, pp. 195-200. Actas del Congreso Internacional FEL XV-PUCE I. Quito, Ecuador: Centro Cultural Puce.

Censabella, Marisa Inés (2009a). Chaco ampliado. En Inge Sichra (ed. y comp.). Atlas sociolingüístico de los pueblos indigenas de América Latina. Tomo I, pp. 143-228. Cochabamba: UNICEF/FUNDPROEIB Andes.

Censabella, Marisa Inés (2009b). Sistema fonológico y sincronía dinámica de seis variedades orientales del continuum wichí. En Ana Fernández Garay; Marisa Censabella (eds.). Estudios fonológicos de continua dialectales: mapuche y wichí, pp. 109-144.Santa Rosa: Universidad Nacional de La Pampa. 
Cleasson, Kenneth (1994). A phonological outline of Mataco-Noctenes. International Journal of American Linguistics 60(1): 1-38.

Fernández Garay, Ana; Spinelli, Silvia (2009). Sincronía dinámica del sistema fonológico del wichí hablado en la Banda Norte del departamento de Rivadavia, Salta. En Ana Fernández Garay; MarisaCensabella (eds.). Estudios fonológicos de continua dialectales: mapuche y wichí, pp. 145-174.Santa Rosa: Universidad Nacional de La Pampa.

Flemming, Edward (2004). Contrast and perceptual distinctiveness. En Bruce Hayes; Robert; Donca Esteriade (eds.). Phonetically based Phonology, pp. 232-276. Cambridge: Cambridge UniversityPress.

Gerzeinsten, Ana (1992). Una variedad oriental del mataco. En Hacia una nueva carta étnica del Gran Chaco $I V$, pp. 67-79. Las Lomitas: Centro del Hombre Antiguo Chaqueño (CHACO).

Avram, Megan Leigh Zdrojkowski (2008). A phonological description of Wichi: the dialect of Misión La Paz, Salta, Argentina. Master's Theses and Doctoral Dissertations. Eastern Michigan University.

Labov, William (1994). General principles of vowel shifting. En William Labov (1994). Principles of linguistic change. Internal factors, pp. 115-155. Oxford: Blackwell.

Messineo, Cristina; Braunstein, José (1990).Variantes lingüísticas del mataco. En Hacia una nueva carta étnica del Gran Chaco, I. pp. 1-13. Las Lomitas: Centro del Hombre Antiguo Chaqueño.

Najlis, Elena (1968). Dialectos del mataco. Anales de la Universidad del Salvador, 4, pp. 5-14. Buenos Aires: Universidad del Salvador.

Najlis, Elena (1984). Fonología de la proto lengua mataguaya. Cuadernos de Lingüística Indígena nº 9 . Buenos Aires: Instituto de Lingüística, Universidad de Buenos Aires.

Nercesián, Verónica (2011). Gramática del Wichí, una lengua chaqueña: Interacción fonología-morfologíasintaxis en el léxico. Tesis de doctorado. Buenos Aires: Universidad de Buenos Aires.

Suárez, María Eugenia; Arenas, Pastor (2102). Plantas y hongos tintóreos de los wichís del Gran Chaco. Boletín de la Sociedad Argentina de Botánica 47 (1/2): 275-283. Disponible en: http://www.scielo.org.ar/scielo. php?script=sci_arttext\&pid=S1851-23722012000100017 Acceso: 10/03/2014.

Terraza, Jimena (2003). Los préstamos en wichí y su aporte al análisis fonético-fonológico. Ponencia presentada en el $51^{\circ}$ Congreso Internacional de Americanistas, Santiago de Chile, 14 al 18 de julio.

Terraza, Jimena (2009). Gramática del wichi: fonología y morfosintaxis. Tesis de doctorado. Canadá: Universidad de Québec en Montreal.

Tovar, Antonio (1984). Catálogo de las lenguas de América del Sur. Madrid: Gredos.

Viñas Urquiza, María Teresa (1974). Lengua mataca. Tomos 1 y 2. Archivo de lenguas precolombinas. Bs. As: Centro de estudios lingüísticos, UBA.

Recebido: 26/11/2014

Versão revista e corrigida: 21/4/2015

Aceito: 30/8/2015. 